\title{
ON THE WELL-POSEDNESS OF THE INITIAL VALUE PROBLEM FOR ELASTIC-PLASTIC OSCILLATORS WITH ISOTROPIC WORK-HARDENING
}

\author{
BY \\ KEMING WANG \\ Department of Mathematics, Case Western Reserve University, Cleveland, Ohio
}

\begin{abstract}
The system of differential equations of elastic-plastic oscillators with isotropic work-hardening is converted to a system of differential inclusions and wellposedness is established using maximal monotone operator theory when the external force $f \in W^{1,1}(0, T ; R)$. By a more delicate analysis, well-posedness is also established for $f \in L^{1}(0, T ; R)$.
\end{abstract}

1. Introduction. In this paper, we study the well-posedness of the system of differential equations for elastic-plastic oscillators with isotropic work-hardening. By converting the system of differential equations to a system of differential inclusions, well-posedness is established using maximal monotone operator theory.

The existence and uniqueness of the system of differential equations for elasticplastic oscillators have been a subject of many studies [1, 2, 3, 4, 5]. [1] used Filippov solutions to show the existence of the solutions for the system of differential equations of elastic-plastic oscillators without work-hardening, and that method can also be applied to the isotropic work-hardening model. By a limiting argument of viscoplastic solutions, [3] established the existence for the material with both kinematic and isotropic work-hardening. [2] and [4] proved the uniqueness of the solutions for the isotropic work-hardening model by different arguments. Some abstract models for elastoplastic systems have been proposed and the well-posedness for those models is established [6, 7]. However, the connections between the model of the elastic-plastic oscillators with isotropic work-hardening and the assumptions of constitutive relations for their abstract models is not clear.

Following the idea of [5], we introduce a new variable that enables us to establish the equivalence of the system of differential equations for elastic-plastic oscillators with isotropic work-hardening and a system of differential inclusions. The operator associated with this system of differential inclusions turns out to be maximal monotone. Hence, the well-posedness of the system of differential inclusions can easily be obtained when the external force $f \in W^{1,1}(0, T ; R)$. By a more delicate analy-

Received January 6, 1993.

1991 Mathematics Subject Classification. Primary 73E50, 34A34.

(C)1995 Brown University 
sis, well-posedness is established when $f \in L^{1}(0, T ; R)$. Therefore, we prove that the system of differential equations for elastic-plastic oscillators with isotropic workhardening is well-posed when $f \in L^{1}(0, T ; R)$. This method can also be applied to other models of elastic-plastic materials, and some properties of the solutions can be discussed by using maximal monotone operator theory.

2. Preliminaries. Let $v, \sigma, f, u^{p}$, and $E$ be the velocity, stress, external force, plastic deformation, and elastic constant, respectively. Let $H:(-\delta, \infty) \rightarrow(0, \infty)$ be a concave strictly increasing function. Here $\delta$ is a positive number.

We consider the following system of differential equations for elastic-plastic oscillators with isotropic work-hardening:

subject to the constraint

$$
(\mathrm{EPO}) \quad\left\{\begin{aligned}
& \dot{v}(t)= f(t)-\sigma(t), \\
& \dot{\sigma}(t)= \begin{cases}\frac{E H^{\prime}\left(\bar{u}^{p}(t)\right)}{E+H^{\prime}\left(\bar{u}^{p}(t)\right)} v(t) & \text { if }|\sigma(t)|=H\left(\bar{u}^{p}(t)\right) \text { and } \sigma(t) v(t) \geq 0 \\
E v(t) & \text { otherwise, }\end{cases} \\
& \dot{u}^{p}(t)= \begin{cases}\frac{E}{E+H^{\prime}\left(\bar{u}^{p}(t)\right)} v(t) & \text { if }|\sigma(t)|=H\left(\bar{u}^{p}(t)\right) \text { and } \sigma(t) v(t) \geq 0 \\
0 & \text { otherwise } \\
& \left(v(0), \sigma(0), u^{p}(0)\right)=\left(v_{0}, \sigma_{0}, u_{0}^{p}\right)\end{cases}
\end{aligned}\right.
$$$$
|\sigma(t)| \leq H\left(\bar{u}^{p}(t)\right),
$$

where

$$
\bar{u}^{p}(t)=\bar{u}^{p}\left(u_{0}^{p}\right)+\int_{0}^{t}\left|\dot{u}^{p}(s)\right| d s .
$$

For simplicity, we assume that the constants $\bar{u}^{p}\left(u_{0}^{p}\right)=0$ and $E=1$.

Definition 1. Let $T>0$. We say that a set of absolutely continuous functions $\left\{\left(v(t), \sigma(t), u^{p}(t)\right)|| \sigma(t) \mid \leq H\left(\bar{u}^{p}(t)\right)\right\}$ is a solution of (EPO) on $[0, T]$ if it satisfies (EPO) for almost every $t \in[0, T]$ and $\left(v(0), \sigma(0), u^{p}(0)\right)=\left(v_{0}, \sigma_{0}, u_{0}^{p}\right)$.

We wish to establish the well-posedness of (EPO). More precisely, we want to show (EPO) has a unique solution that depends continuously on the given data, i.e., on the external force and initial values.

Since the function $H\left(\bar{u}^{p}\right)$ is monotonically increasing, we can define a one-to-one transformation by

$$
\hat{u}^{p}\left(\bar{u}^{p}\right)=\int_{0}^{\bar{u}^{p}} \sqrt{H^{\prime}(s)} d s
$$

and introduce a new function $G\left(\hat{u}^{p}\right)$ such that

$$
G(0)=H(0) \quad \text { and } \quad G^{\prime}\left(\hat{u}^{p}\right)=\sqrt{H^{\prime}\left(\bar{u}^{p}\right)} .
$$


Hence, for each $\hat{u}_{a}^{p}=\hat{u}^{p}\left(\bar{u}_{a}^{p}\right)$ it follows that

$$
\begin{aligned}
G\left(\hat{u}_{a}^{p}\right) & =\int_{0}^{\hat{u}_{a}^{p}} G^{\prime}\left(\hat{u}^{p}\right) d \hat{u}^{p}+G(0) \\
& =\int_{0}^{\tilde{u}_{a}^{p}} \sqrt{H^{\prime}\left(\bar{u}^{p}\right)} \sqrt{H^{\prime}\left(\bar{u}^{p}\right)} d \bar{u}^{p}+H(0)=H\left(\bar{u}_{a}^{p}\right),
\end{aligned}
$$

and we have

$$
G\left(\hat{u}^{p}\right)=H\left(\bar{u}^{p}\right) .
$$

It is easy to see that the function $G:\left(-\delta_{0}, \infty\right) \rightarrow(0, \infty)$ is concave strictly increasing, where $\delta_{0}=\int_{-\delta}^{0} \sqrt{H^{\prime}(s)} d s$.

It follows from (3), (4), and (5) that

$$
\dot{\hat{u}}^{p}(t)=\sqrt{H^{\prime}\left(\bar{u}^{p}(t)\right)} \dot{\bar{u}}^{p}(t)=G^{\prime}\left(\hat{u}^{p}(t)\right)\left|\dot{u}^{p}(t)\right|=G^{\prime}\left(\hat{u}^{p}(t)\right) \dot{u}^{p}(t) \operatorname{Sign}(\sigma(t)),
$$

where

$$
\operatorname{Sign}(\sigma(t)):= \begin{cases}1 & \text { if } \sigma(t)>0 \\ 0 & \text { if } \sigma(t)=0 \\ -1 & \text { if } \sigma(t)<0\end{cases}
$$

By (5) and (EPO), (8) yields

$$
\dot{\hat{u}}^{p}(t)= \begin{cases}\frac{G^{\prime}\left(\hat{u}^{p}(t)\right)}{1+G^{2}\left(\hat{u}^{p}(t)\right)} v(t) \operatorname{Sign}(\sigma(t)) & \text { if }|\sigma(t)|=G\left(\hat{u}^{p}(t)\right) \text { and } \sigma(t) v(t) \geq 0, \\ 0 & \text { otherwise, }\end{cases}
$$

where we have used $E=1$. Therefore, we get the following lemma.

LEMMA 1. (EPO) is equivalent to the following system of differential equations:

$$
(\overline{\mathrm{EPO}})\left\{\begin{array}{l}
\dot{v}(t)=f(t)-\sigma(t), \\
\dot{\sigma}(t)= \begin{cases}\frac{G^{\prime 2}\left(\hat{u}^{p}(t)\right)}{1+G^{\prime 2}\left(\hat{u}^{p}(t)\right)} v(t) & \text { if }|\sigma(t)|=G^{\prime}\left(\hat{u}^{p}(t)\right) \quad \text { and } \sigma(t) v(t) \geq 0, \\
v(t) & \text { otherwise, }\end{cases} \\
\dot{\hat{u}}^{p}(t)=\left\{\begin{array}{ll}
\frac{G^{\prime}\left(\hat{u}^{p}(t)\right)}{1+G^{\prime 2}\left(\hat{u}^{p}(t)\right)} v(t) \operatorname{Sign}(\sigma(t)) \text { if }|\sigma(t)|=G\left(\hat{u}^{p}(t)\right), \sigma(t) v(t) \geq 0, \\
0 \quad & \text { otherwise, }
\end{array},\left(v(0), \sigma(0), \hat{u}^{p}(0)\right)=\left(v_{0}, \sigma_{0}, 0\right),\right.
\end{array}\right.
$$

subject to the constraint

$$
|\sigma(t)| \leq G\left(\hat{u}^{p}(t)\right),
$$

where $G:\left(-\delta_{0}, \infty\right) \rightarrow(0, \infty)$ is a concave strictly increasing function.

3. Main results. We define

$$
C:=\left\{\left(\sigma, \hat{u}^{p}\right) \in R \times\left(-\delta_{0}, \infty\right)|| \sigma \mid \leq G\left(\hat{u}^{p}\right)\right\} .
$$


The indicator function $I_{c}$ of the subset $C$ of $R^{2}$ is defined by

$$
I_{c}\left(\sigma, \hat{u}^{p}\right)= \begin{cases}0 & \text { if }\left(\sigma, \hat{u}^{p}\right) \in C, \\ +\infty & \text { if }\left(\sigma, \hat{u}^{p}\right) \in R^{2} \backslash C .\end{cases}
$$

$I_{c}$ is proper convex and lower semicontinuous and the multivalued subgradient $\partial I_{c}$ of $I_{c}$ is

$$
\partial I_{c}\left(\sigma, \hat{u}^{p}\right)= \begin{cases}\lambda\left(\operatorname{Sign}(\sigma),-G^{\prime}\left(\hat{u}^{p}\right)\right), & \text { if }|\sigma|=G\left(\hat{u}^{p}\right), \\ (0,0) & \text { if }|\sigma|<G\left(\hat{u}^{p}\right),\end{cases}
$$

where $\lambda$ is a positive number.

Let us consider the following system of differential inclusions:

$$
\text { (DI) } \quad\left\{\begin{array}{l}
\dot{v}(t)=f(t)-\sigma(t), \\
\left(\dot{\sigma}(t), \dot{\hat{u}}^{p}(t)\right) \in(v(t), 0)-\partial I_{c}\left(\sigma(t), \hat{u}^{p}(t)\right)
\end{array}\right.
$$

with initial condition

$$
\left(v(0), \sigma(0), \hat{u}^{p}(0)\right)=\left(v_{0}, \sigma_{0}, 0\right) .
$$

Definition 2. Let $T>0$. We say that a set of absolutely continuous functions $\left(v(t), \sigma(t), \hat{u}^{p}(t)\right)$ is a solution of (DI) on $[0, T]$ if it satisfies (DI) for almost every $t \in[0, T]$ and $\left(v(0), \sigma(0), \hat{u}^{p}(0)\right)=\left(v_{0}, \sigma_{0}, 0\right)$.

THEOREM 1. (EPO) is equivalent to (DI).

Proof. It suffices to show that (DI) and ( $\overline{\mathrm{EPO}})$ are equivalent by Lemma 1.

First, we want to show that the solution of $(\overline{\mathrm{EPO}})$ is a solution of (DI). Let $\left(u(t), \sigma(t), \hat{u}^{p}(t)\right)$ be the solution of the $(\overline{\mathrm{EPO}})$. Let

$$
E_{t}:=\left\{t \in[0, T]|| \sigma(t) \mid<G\left(\hat{u}^{p}(t)\right)\right\}
$$

and

$$
P_{t}:=\left\{t \in[0, T]|| \sigma(t) \mid=G\left(\hat{u}^{p}(t)\right)\right\} .
$$

When $|\sigma(t)|<G\left(\hat{u}^{p}(t)\right)$, it follows from $(\overline{\mathrm{EPO}})$ that for a.e. $t \in E_{t}$

$$
\dot{\sigma}(t)=v(t) \quad \text { and } \quad \dot{\hat{u}}^{p}(t)=0 .
$$

Hence,

$$
\left(\dot{\sigma}(t)-v(t), \dot{\hat{u}}^{p}(t)\right)=(0,0)=\partial I_{c}\left(\sigma(t), \hat{u}^{p}(t)\right)
$$

for a.e. $t \in E_{t}$ and (DI) is satisfied.

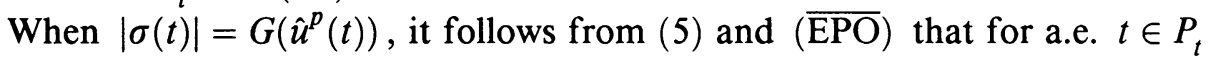

$$
\dot{\hat{u}}^{p}(t)=-G^{\prime}\left(\hat{u}^{p}(t)\right) \dot{\vec{u}}^{p}(t)
$$

and

$$
v(t)-\dot{\sigma}(t)=\operatorname{Sign}(\sigma(t)) \dot{\bar{u}}^{p}(t)
$$

for both cases $\sigma(t) v(t) \geq 0$ and $\sigma(t) v(t)<0$. Therefore, for a.e. $t \in P_{t}$,

$$
\left(v(t)-\dot{\sigma}(t),-\dot{\hat{u}}^{p}(t)\right) \in \lambda\left(\operatorname{Sign}(\sigma(t)),-G^{\prime}\left(\hat{u}^{p}\right)(t)\right)=\partial I_{c}\left(\sigma(t), \hat{u}^{p}(t)\right) .
$$

Hence, (DI) is satisfied. 
Next, we want to show that the solution of $(\mathrm{DI})$ is a solution of $(\overline{\mathrm{EPO}})$. Let $\left(u(t), \sigma(t), \hat{u}^{p}(t)\right)$ be the solution of (DI).

When $t \in E_{t}$, we have $|\sigma(t)|<G\left(\hat{u}^{p}(t)\right)$ and for a.e. $t \in E_{t}$

$$
\dot{\sigma}(t)=v(t) \quad \text { and } \quad \dot{\hat{u}}^{p}(t)=0
$$

by (DI).

When $t \in P_{t}$, we have $|\sigma(t)|=G\left(\hat{u}^{p}(t)\right)$ and for a.e. $t \in P_{t}$

$$
\operatorname{Sign}(\sigma(t)) \dot{\sigma}(t)=G^{\prime}\left(\hat{u}^{p}(t)\right) \dot{\hat{u}}^{p}(t)
$$

by differentiating both sides with respect to $t$. It follows from (DI) that

$$
\left(v(t)-\dot{\sigma}(t),-\dot{\hat{u}}^{p}(t)\right)=\left(\operatorname{Sign}(\sigma(t)),-G^{\prime}\left(\hat{u}^{p}(t)\right)\right) \lambda
$$

for a.e. $t \in P_{t}$; here $\lambda \geq 0$. Therefore, we have

$$
\operatorname{Sign}(\sigma(t)) \dot{\sigma}(t)=\lambda\left(G^{\prime}\left(\hat{u}^{p}(t)\right)\right)^{2}
$$

and

$$
0 \leq \lambda=\frac{1}{G^{2}} \operatorname{Sign}(\sigma(t)) \dot{\sigma}(t) .
$$

for a.e. $t \in P_{t}$. It follows from (20) and (22) that

$$
\left(v(t)-\dot{\sigma}(t),-\dot{\hat{u}}^{p}(t)\right)=\left(\frac{\dot{\sigma}(t)}{G^{\prime 2}},-\frac{1}{G^{\prime}} \operatorname{Sign}(\sigma(t)) \dot{\sigma}(t)\right),
$$

and $\operatorname{Sign}(\sigma(t)) \dot{\sigma}(t) \geq 0$ for a.e. $t \in P_{t}$. By solving $\dot{\sigma}(t)$ from (23), we get

$$
\begin{aligned}
\dot{\sigma}(t) & =\frac{G^{2}\left(\hat{u}^{p}(t)\right)}{1+G^{\prime 2}\left(\hat{u}^{p}(t)\right)} v(t), \\
\dot{\hat{u}}^{p}(t) & =\frac{G^{\prime}\left(\hat{u}^{p}(t)\right)}{1+G^{\prime 2}\left(\hat{u}^{p}(t)\right)} v(t) \operatorname{Sign}(\sigma(t)),
\end{aligned}
$$

and $\sigma(t) v(t) \geq 0$ for a.e. $t \in P_{t}$.

By (19) and (24), we obtain for a.e. $t \in[0, T]$

$$
\begin{aligned}
\dot{\sigma}(t) & = \begin{cases}\frac{G^{\prime 2}\left(\hat{u}^{p}(t)\right)}{1+G^{\prime 2}\left(\hat{u}^{p}(t)\right)} v(t) & \text { if }|\sigma(t)|=G^{\prime}\left(\hat{u}^{p}(t)\right) \text { and } \sigma(t) v(t) \geq 0, \\
v(t) & \text { otherwise, }\end{cases} \\
\dot{\hat{u}}^{p}(t) & = \begin{cases}\frac{G^{\prime}\left(\hat{u}^{p}(t)\right)}{1+G^{\prime 2}\left(\hat{u}^{p}(t)\right)} v(t) \operatorname{Sign}(\sigma(t)) & \text { if }|\sigma(t)|=G\left(\hat{u}^{p}(t)\right) \text { and } \sigma(t) v(t) \geq 0, \\
0 & \text { otherwise. }\end{cases}
\end{aligned}
$$

Since the domain of $\partial I_{c}$ is the set $C$ and the solution of (DI) lies in the domain of $\partial I_{c}$, we have $|\sigma(t)| \leq G\left(\hat{u}^{p}(t)\right)$ for all $t \in[0, T]$. Hence, $\left(u(t), \sigma(t), \hat{u}^{p}(t)\right)$ is a solution of $(\overline{\mathrm{EPO}})$.

Now, we want to apply the maximal monotone operator theory to show the wellposedness of (DI). 
THEOREM 2. Let $T>0$ be given and let $f \in W^{1,1}(0, T ; R)$. (DI) has a unique solution that depends continuously on given data if $\left(\sigma_{0}, \hat{u}_{0}^{p}\right) \in C$.

Proof. Let $A$ be an operator that maps $\left(v, \sigma, \hat{u}^{p}\right)$ to $(\sigma,-v, 0)$. Let

$$
\bar{C}:=\left\{\left(v, \sigma, \hat{u}^{p}\right) \in R \times R \times\left(-\delta_{0}, \infty\right)|| \sigma \mid \leq G\left(\hat{u}^{p}\right)\right\}
$$

and let $I_{\bar{c}}$ be the indicator function of $\bar{C}$.

Denote $x(t)=\left(v(t), \sigma(t), \hat{u}^{p}(t)\right)$. (DI) can be written as

$$
\dot{x}(t)+\left(A+\partial I_{\tilde{z}}\right) x(t)=\bar{f}(t),
$$

where $\bar{f}(t)=(f(t), 0,0)$.

Since $\bar{C}$ is a convex closed subset of $R^{3}, \partial I_{c}$ is a maximal monotone operator. Because $A$ is a continuous and everywhere defined monotone operator, $A+\partial I_{\bar{\tau}}$ is also a maximal monotone operator by Theorem 3.2 of [8] (page 158). Since $\left(\sigma_{0}, \hat{u}_{0}^{p}\right)$ is in the domain of $\partial I_{n}$, we have $\left(v_{0}, \sigma_{0}, \hat{u}_{0}^{p}\right)$ is in the domain of $A+\partial I_{\tilde{c}}$. Hence, (DI) is well-posed [9].

Next, we want to weaken the regularity of $f$. More precisely, we wish to show the well-posedness of (DI) when $f \in L^{1}(0, T ; R)$.

Lemma 2 (see [10]). Let $m \in L^{1}(0, T ; R)$ be such that $m(t) \geq 0$ for a.e. $t \in(0, T)$ and $b \geq 0$ a constant. Let $\phi:[0, T] \rightarrow R$ be a continuous function that satisfies

$$
\phi^{2}(t) \leq b^{2}+2 \int_{0}^{t} m(s) \phi(s) d s \quad \forall t \in[0, T] .
$$

Then

$$
|\phi(t)| \leq b+\int_{0}^{t} m(s) d s \quad \forall t \in[0, T] .
$$

Theorem 3. Let $f_{i} \in L^{1}(0, T ; R), i=1,2$ and let $\left(v_{i}(t), \sigma_{i}(t), \hat{u}_{i}^{p}(t)\right)$ be a solution of (DI) with initial condition $\left(v_{i}(0), \sigma_{i}(0), \hat{u}_{i}^{p}(0)\right)$ and external force $f_{i}$. Then

$$
\begin{aligned}
& \left(\left|v_{1}-v_{2}\right|+\left|\sigma_{1}-\sigma_{2}\right|+\left|\hat{u}_{1}^{p}-\hat{u}_{2}^{p}\right|\right)(t) \\
& \quad \leq\left(\left|v_{1}-v_{2}\right|+\left|\sigma_{1}-\sigma_{2}\right|+\left|\hat{u}_{1}^{p}-\hat{u}_{2}^{p}\right|\right)(0)+\int_{0}^{t}\left|f_{1}(s)-f_{2}(s)\right| d s .
\end{aligned}
$$
have

Proof. Denote $x_{i}(t)=\left(v_{i}(t), \sigma_{i}(t), \hat{u}_{i}^{p}(t)\right)$. By the monotonicity of $A+\partial I_{\bar{c}}$ we

$$
\left\langle\dot{x}_{1}(t)-\dot{x}_{2}(t), x_{1}(t)-x_{2}(t)\right\rangle \leq\left\langle f_{1}(t)-f_{2}(t), x_{1}(t)-x_{2}(t)\right\rangle,
$$

where $\langle$,$\rangle stands for inner product. Hence,$

$$
\left(x_{1}-x_{2}\right)^{2}(t) \leq\left(x_{1}-x_{2}\right)^{2}(0)+2 \int_{0}^{t}\left|\left(f_{1}(s)-f_{2}(s)\right) \|\left(x_{1}(s)-x_{2}(s)\right)\right| d s .
$$

Therefore,

$$
\left(x_{1}-x_{2}\right)(t) \leq\left(x_{1}-x_{2}\right)(0)+\int_{0}^{t}\left|\left(f_{1}(s)-f_{2}(s)\right)\right| d s
$$

by Lemma 2 . 
Theorem 4. Let $f \in L^{1}(0, T ; R)$. (DI) has a solution if $\left(\sigma_{0}, \hat{u}_{0}^{p}\right) \in C$.

Proof. Let $n \in N$ and $\left(\partial I_{\bar{c}}\right)_{n}$ be a sequence of Yosida approximation of $\partial I_{\bar{c}}$. Since $\left(v_{0}, \sigma_{0}, \hat{u}_{0}^{p}\right)$ is in the domain of $\partial I_{\bar{c}}$ and the domain of $\partial I_{\bar{c}}$ is a subset of the domain of $\left(\partial I_{\bar{c}}\right)_{n}$ for each $n \in N,\left(v_{0}, \sigma_{0}, \hat{u}_{0}^{p}\right)$ is in the domain of $\left(\partial I_{\bar{c}}\right)_{n}$ for each $n \in N$. Because $\left(\partial I_{\tilde{c}}\right)_{n}$ is Lipschitz continuous and $f \in L^{1}(0, T ; R)$, the following equation has a unique solution for each $n \in N$ :

$$
\dot{x}_{n}(t)+\left(A+\left(\partial I_{\bar{c}}\right)_{n}\right) x_{n}(t)=\bar{f}(t)
$$

with initial condition

$$
x_{n}(0)=\left(v_{0}, \sigma_{0}, \hat{u}_{0}^{p}\right),
$$

where $\bar{f}(t)=(f(t), 0,0)$.

Since

$$
\left(\partial I_{\bar{c}}\right)_{n} x=\frac{1}{n}\left(x-\operatorname{Proj}_{\bar{c}} x\right),
$$

we have $\left(\partial I_{\tilde{c}}\right)_{n} 0=0$. Hence, for a.e. $t \in[0, T]$

$$
\left\langle\left(A+\left(\partial I_{\bar{c}}\right)_{n}\right) x_{n}(t), x_{n}(t)\right\rangle=\left\langle\left(A+\left(\partial I_{\bar{c}}\right)_{n}\right) x_{n}(t)-\left(A+\left(\partial I_{\bar{c}}\right)_{n}\right) 0, x_{n}(t)-0\right\rangle \leq 0 .
$$

It follows from (29) and (31) that for a.e. $t \in[0, T]$

$$
\left\langle\dot{x}_{n}(t), x_{n}(t)\right\rangle \leq\left\langle\bar{f}(t), x_{n}(t)\right\rangle \text {. }
$$

Therefore,

$$
x_{n}^{2}(t) \leq x_{n}^{2}(0)+\int_{0}^{t}|\bar{f}(s)|\left|x_{n}(s)\right| d s \leq x^{2}(0)+\int_{0}^{T}|\bar{f}(s)|\left|x_{n}(s)\right| d s
$$

for each $n \in N$ and $t \in[0, T]$. Thus, $x_{n}(t)$ is uniformly bounded on $[0, T]$ by Lemma 2.

Since for a.e. $t \in[0, T]$

$$
\left(\dot{\sigma}_{n}(t), \dot{\hat{u}}_{n}^{p}(t)\right)=\left(v_{n}(t), 0\right)-\partial_{n} I_{c}\left(\sigma_{n}(t), \hat{u}_{n}^{p}(t)\right),
$$

we have

$$
\begin{aligned}
\left\langle\left(\dot{\sigma}_{n}(t), \dot{\hat{u}}_{n}^{p}(t)\right),\left(\dot{\sigma}_{n}(t), \dot{\hat{u}}_{n}^{p}(t)\right)\right\rangle+\left\langle\partial_{n} I_{c}\left(\sigma_{n}(t), \hat{u}_{n}^{p}(t)\right),\right. & \left.\left(\dot{\sigma}_{n}(t), \dot{\hat{u}}_{n}^{p}(t)\right)\right\rangle \\
= & \left\langle\left(v_{n}(t), 0\right),\left(\dot{\sigma}_{n}(t), \dot{\hat{u}}_{n}^{p}(t)\right)\right\rangle
\end{aligned}
$$

for a.e. $t \in[0, T]$. It follows from (33) that for each $n \in N$

$$
\int_{0}^{T}\left(\dot{\sigma}_{n}^{2}(s)+\left(\dot{\hat{u}}_{n}^{p}\right)^{2}(s)\right) d s+\left(I_{c}\right)_{n}\left(\sigma_{n}(T), \hat{u}_{n}^{p}(T)\right)-\left(I_{c}\right)_{n}\left(\sigma_{n}(0), \hat{u}_{n}^{p}(0)\right)=\int_{0}^{T} v_{n}(s) \dot{\sigma}_{n}(s) d s,
$$

where

$$
\left(I_{c}\right)_{n}(x)=\frac{1}{2 n}\left|x-\operatorname{Proj}_{c} x\right|^{2} .
$$

Since $\left(\sigma_{n}(0), \hat{u}_{n}^{p}(0)\right)=\left(\sigma_{0}, \hat{u}_{0}^{p}\right) \in C$, we have

$$
\left(I_{c}\right)_{n}\left(\sigma_{n}(0), \hat{u}_{n}^{p}(0)\right)=0 \text { and }\left(I_{c}\right)_{n}\left(\sigma_{n}(T), \hat{u}_{n}^{p}(T)\right) \geq 0 .
$$

Hence, (34) yields

$$
\int_{0}^{T}\left(\dot{\sigma}_{n}^{2}(s)+\left(\dot{\hat{u}}_{n}^{p}\right)^{2}(s)\right) d s \leq\left(\int_{0}^{T}\left|v_{n}(s)\right|^{2} d s\right)^{1 / 2}\left(\int_{0}^{T}\left|\dot{\sigma}_{n}(s)\right|^{2} d s\right)^{1 / 2}
$$


for each $n \in N$. We conclude that the $L^{2}$ norms of $\dot{\sigma}_{n}, \dot{\hat{u}}_{n}^{p}$ are uniformly bounded by the uniform boundedness of $v_{n}$. Therefore, the $L^{1}$ norms of $\dot{\sigma}_{n}, \dot{\hat{u}}_{n}^{p}$ are also uniformly bounded.

Since $f(t) \in L^{1}(0, T ; R)$ and

$$
\dot{v}_{n}(t)=\sigma_{n}(t)+f(t),
$$

the $L^{1}$ norm of $\dot{v}_{n}$ is uniformly bounded. Hence, $\left(v_{n}, \sigma_{n}, \hat{u}_{n}^{p}\right)$ converges uniformly on $[0, T]$ to a set $\left(v, \sigma, \hat{u}^{p}\right)$ of absolutely continuous functions. Moreover, $\left(\dot{v}_{n}, \dot{\sigma}_{n}, \dot{\hat{u}}_{n}^{p}\right)$ converges weakly to $\left(\dot{v}, \dot{\sigma}, \dot{\hat{u}}^{p}\right)$ in $L^{1}(0, T ; R)$. Therefore, $\left(\dot{v}, \dot{\sigma}, \dot{\hat{u}}^{p}\right)$ is the solution of (DI) by the demiclosedness of the maximal monotone operator $A+\partial I_{\bar{c}}$.

It follows from Theorems 1,3 , and 4 that the following theorem holds.

THEOREM 5. Let $f \in L^{1}(0, T ; R)$. (EPO) has a unique solution that depends continuously on initial data and the external force if $\left|\sigma_{0}\right| \leq H\left(\bar{u}^{p}(0)\right)$.

Acknowledgment. I would like to thank Professor D. R. Owen for helpful discussions.

\section{REFERENCES}

[1] J. L. Buhite and D. R. Owen, An ordinary differential equation from the theory of plasticity, Arch. Rational Mech. Anal. 71, 357-383 (1979)

[2] D. R. Owen, Weakly decaying energy separation and uniqueness of motions of an elastic-plastic oscillator with work-hardening, Arch. Rational Mech. Anal. 98, 95-114 (1987)

[3] M. M. Suliciu, I. Suliciu, and W. Williams, On viscoelastic-plastic oscillators, Quart. Appl. Math. 47, 105-116 (1989)

[4] D. R. Owen and K. Wang, Weakly Lipschitzian mappings and restricted uniqueness of solutions of ordinary differential equations, J. Differential Equations 95, 385-398 (1992)

[5] T. Miyoshi, Foundations of the numerical analysis of plasticity, North-Holland, 1985

[6] K. Gröger, Evolution equations in the theory of plasticity, Proc. Fifth Summer School on Nonlinear Operators, Berlin, 1977

[7] K. Gröger, J. Nečas, and L. Trávníček, Dynamic deformation processes of elastic-plastic systems, ZAMM 59, 567-572 (1979)

[8] V. Barbu, Nonlinear semigroups and differential equations in Banach spaces, Noordhoff, Leyden, 1976

[9] E. Zeidler, Nonlinear functional analysis and its applications, Vol. II/A, Springer-Verlag, New York, 1990

[10] H. Brêzis, Opérateurs maximaux monotones et semi-groupes de contractions dans les espaces de Hilbert, Math. Studies 5, North Holland, 1973 